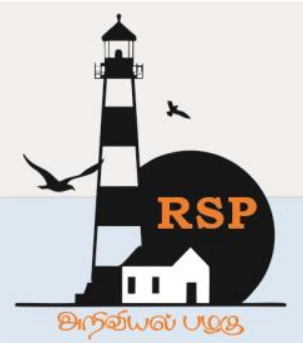

INTERNATIONAL RESEARCH JOURNAL ON ADVANCED SCIENCE HUB

RSP SCIENCE HUB

(The Hub of Research Ideas)

Available online at www.rspsciencehub.com

\title{
Investigation on the Synthesis and Chemical Properties of Nanomaterials
}

E. Bhawani ${ }^{1}$, Vivek Kumar ${ }^{2}$, T. Babu ${ }^{3}$, Balgovind Tiwari ${ }^{* 4}$, R.N.P. Choudhary ${ }^{5}$

${ }^{1}$ Mentor, Dept. of Physics, IIIT-RKValley, RGUKT, A.P., India

${ }^{2}$ Assistant Professor, Dept. of Chemical Engineering, IIIT-RKValley, RGUKT, A.P., India

${ }^{3}$ Teacher, Dept. of Physics, Sri Chaitanya High School, Vempalli, Kadapa, A.P., India

${ }^{4}$ Assistant Professor, Dept. of Physics, IIIT-RKValley, RGUKT, A.P., India

${ }^{5}$ Professor, Dept. of Physics, ITER-SOA University, Bhubaneswar, Orissa, India

balgovindtiwari@gmail.com ${ }^{4}$

\begin{abstract}
Nanoscience is assumed to have capability to change the efficiency of the current electronic and electrical devices. So nanotechnology is being developed to achieve the same. Various kinds of nanomaterials are being prepared such that they show tremendous properties at the nanoscale dimensions. Hence, it is worth studying about the synthesis of nanomaterials. The information that we present in this paper corresponds to a basic review on the synthesis and recent work done on nanomaterials. The distinctness of this review is that, the classification and major types of nanomaterials has been reported. The literature was collected through internet, from various available sources. The synthesis methods that have been discussed are sputtering, hydrothermal, microwave assisted, and electrochemical and chemical reduction methods.
\end{abstract}

Keywords:Materials; Chemical Engineering; Polymers; Nanomaterials;

\section{Introduction}

Nanoscience and nanotechnology are the terms that have got immense importance in research and development, across the world. There is no precise definition for the terms mentioned above. But the term nanotechnology, may be defined as, " $a$ technical process that involves the preparation and characterization of materials either by reducing their size to nanoscale or using a nano dimensional material" [1]. Nanos, a Greek word, is actually the basis of nano. The Greek word nanos refer to low size or dwarf. The specialty of this particular field is that, it makes the physicists, chemists, solid state scientists, bio-scientists, material scientists, etc. to work together. Because, all the knowledge together contribute to the critical and cutting edge development of nanomaterials. In each and every part of science and technology, nano has got its own importance. Also, it is very important to understand the fundamental difference between nanoscience and nanotechnology [2].

\subsection{Basic Terminology}

Nanoscience: Investigation of arrangement of atoms and their basic properties at the nano level.

Nanotechnology:Developing the novel nanomaterials for devices, by manipulating the properties of nanomaterials at the atomic level.

Nanomaterial: A material with the size of less than 100 nanometers, for at least one dimension.

\subsection{Classification of Nanomaterials}

On the basis of size of nano level dimension i.e. < 100 nanometer, nanomaterials are categorized into different categories $[1,3]$ as shown in figure 1 .

0-dimensional: The nanomaterials of all dimensions in nano level. Nanoparticles are the exception in this category.

1-dimensional: The nanomaterials of atleast one dimension in nano level. Other two dimensions will be in any other level. Examples include rods/wires/tubes of nano type.

2-dimensional: The nanomaterials of atleast two dimensions in nano level. Remaining dimension can be in any other level. Examples include films/layers/coatings of nano type.

3-dimensional: The nanomaterials of every 


\section{Classification of Nanomaterials \\ 0- Dimensional \\ 1- Dimensional \\ 2- Dimensional \\ 3- Dimensional}

\section{Fig. 1. Classification of Nanomaterials}

\subsection{Types of Nanomaterials}

Based on the size and properties, nanomaterials can be categorized into different types as shown in figure 2 .

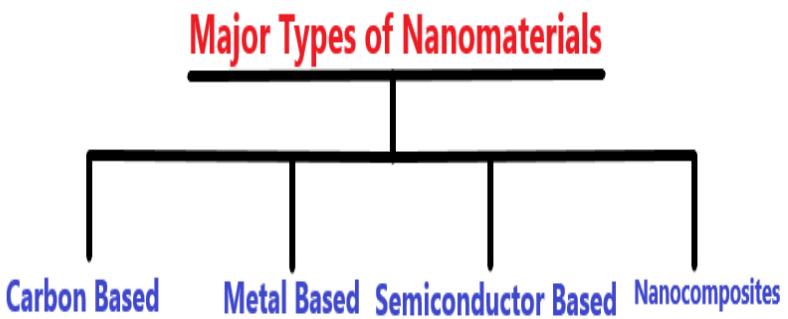

\section{Fig. 2. Types of Nanomaterials}

\subsubsection{Carbon Nanomaterials}

The nanomaterial that contain carbon as the one of its major component, is known as carbon based nanomaterial. Fullerenes and carbon nanotubes come under this category. The carbon nanotubes are incorporated in graphene sheets, and then sheets are rolled into a tube. The mechanical strength of these tubes is very much higher than steel. Fullerenes are the allotropes of carbon [2-3].

\subsubsection{Metal Nanomaterials}

Metal ions of +2 and +3 valency are used as the raw materials to prepare metal based nanoparticles. Preparation can be done through chemical synthesis or photochemical reaction. These metal ions are simplified into metal based nanoparticles, with the help of chemical reductants. The resulting nanoparticles contain large surface areas. Also, it is possible to prepare the new nanoparticles by combining more than two single nanoparticles [23].

\subsubsection{Semiconductor based Nanomaterials}

These kind of nanomaterials are extensively used in catalysis and some electrical applications. Some of the most widely investigated semiconductor nanomaterials are zinc sulphide $(\mathrm{ZnS}), \mathrm{ZnO}$, cadmium sulphide (CdS), cadmium selenide (CdSe), etc. They exhibit both metallic and nonmetallic characteristics. Their properties can be finely tuned by using graphene [1].

\subsubsection{Nanocomposite Materials}

Nanocomposite is a solid substance that possess different phases where at least one phase size should be less than 100 nanometers. Nanocomposites can be further divided into ceramic/metal/polymer matrix composites. These materials has got significant importance in solar cells, and catalysis applications [1].

During the period of earlier decades, development of nanoscience and nanotechnology is exceptionally well, and it should be acknowledged. Distinct nanomaterials having 1-/2-/3-dimension(s) have been developed for the applications such as sensors (both electrical and bio), energy devices (storage and harvesting), catalytic applications, etc. [4-5]. In the technology of sensors and energy devices, nanomaterials of following characteristics are essentially required:

- Large porosity

- High surface energy

- Large surface area (specific surface area)

Because, the above properties hold a key role in the chemical processes occurring at surfaces and interfaces.

Transition metal oxide based nanomaterials exhibit the following features that are quite useful in the applications of electrochemistry [4]. 
- Catalytic activity

- Easy reinforcement in noble metal nanoparticles

- Large abundancy

- High energy storage capacity

- Non-degradation for long time

- Low cost preparation

- Less toxic in nature

One of the most widely used nanomaterial is zinc oxide $(\mathrm{ZnO})$, for several optical and piezoelectric applications. The bandgap of $\mathrm{ZnO}$ nanomaterial is nearly $3.37 \mathrm{eV}$. Nanodevices fabrication, became effective, with the use of $\mathrm{ZnO}$ nanomaterials in the form of spheres, rods, wires, arrays, etc. Because they show good piezoelectric, dielectric, and optical properties. The incorporation of transition metals, in $\mathrm{ZnO}$ nanostructures, resulted in tremendous electrical properties that could be useful for devices based on spin [6]. Also, the magnetic properties can be induced and altered with the introduction of cation of magnetic nature. For instance, chromium $(\mathrm{Cr})$ doped $\mathrm{ZnO}$ exhibit the good magnetic properties. $\mathrm{Cr}$ is identified as one of the suitable modifier for $\mathrm{ZnO}$, for following reasons [7-8]:

- Stable ferromagnetism in Cr-doped $\mathrm{ZnO}$

- Low ionic radius than $\mathrm{Zn}$

- Can easily replace $\mathrm{Zn}$ position

- Paramagnetic nature at high temperatures

- Antiferromagnetic nature at low temperatures

In this review paper, we present a basic understanding on the different preparation methods of nanomaterials, and the recent work done.

\section{Discussion and Analysis}

This section deals with the different preparation methods of nanomaterials of different kinds.

Nanomaterials based on iridium metal, are quite useful in electrical/optical/catalytic applications. Hence, it is worth to have basic understanding on the preparation techniques (majority techniques are shown in figure 3 ) of Iridium based nanomaterials.

\subsection{Preparation through Sputtering}

Sputtering is one of the deposition technique that can be done physically i.e. sputtering is a physical vapor deposition technique. Under the subject of an external and alternating electric field, the charged ions can be accelerated in a specified direction, through sputtering. When these charged ions collide with the target surface, atomization of surface takes place. Then the substrate surface will be sputtered with the neutral atoms, and hence, nano (particles or films) materials are said to be formed. Through this method, nanomaterials of relatively high melting points can be produced. Also, nanocomposites can produced with the usage of multiple sputtering targets. Moreover, the narrow size distribution of nanoparticles can be achieved through this method. Niwa and other researchers, realized an electrode made of Iridium/carbon nanocomposite [9]. It has been identified that the Iridium nanoparticles, which are dispersed on carbon nanofilm, exhibit a size of nearly 2 nanometers. Hence, the surface morphology can be altered/controlled with sputtering method.

\subsection{Electrochemical Preparation}

This method is based on redox reaction. Under the application of electric potential, the dissolved cations (metallic) get discharged, and become crystals on the cathode. With the control of voltage and current, the physical properties of nanomaterials can be disciplined. Through this method, single nanocrystalline materials can be produced on the single crystalline substrates. Using the iridium chloride ( $\mathrm{IrCl}_{3}$-solution type) electrolyte, Iridium nanoparticles are electrochemically deposited. Deposition has been done on the film of carbon nanotube [10]. On average, the size of nanoparticle is approximately around 100 to 180 nanometers [10].

\subsection{Hydrothermal Preparation}

Under the elevated high temperature and pressure conditions, aqueous preparation of nanomaterials is generally referred as hydrothermal method. The preparation is done in a separate closed system where environment pollution is avoided. But still, observing the nanocrystals is quite difficult. Because, the reactions will not stop at high pressure. Nanoparticles based on Iridium, have been prepared through hydrothermal synthesis, in hydroxyethyl piperazineethanesulphonic acid. The temperature condition is $180{ }^{\circ} \mathrm{C}$ [11]. 


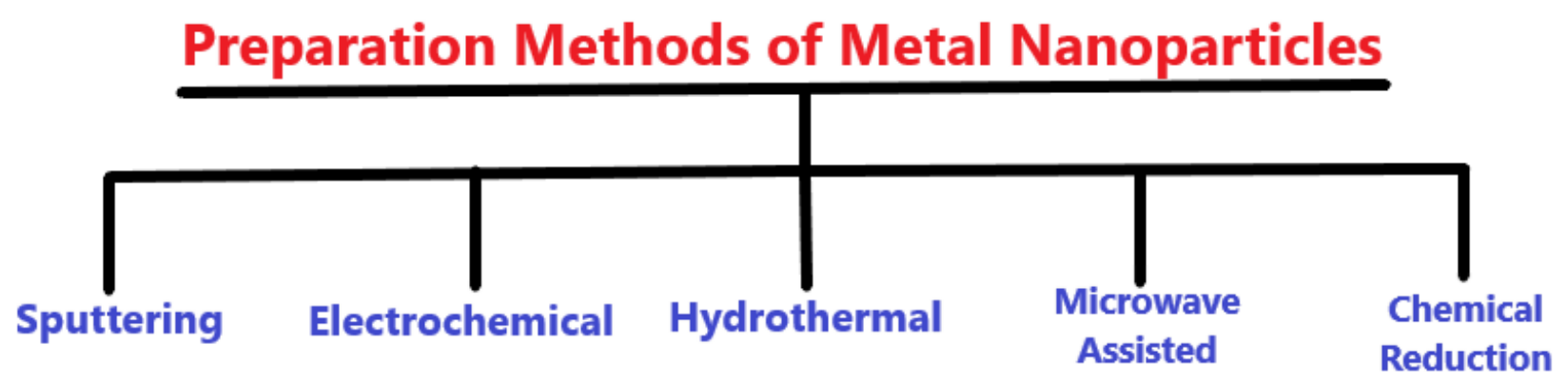

\section{Fig. 3. Different Preparation Techniques of Metal Nanoparticles}

\subsection{Microwave assisted Preparation}

It is one of the rapid technique to produce materials of nanoscale. In the span of very few minutes, nanomaterials can be prepared effectively and efficiently. The frequency (electromagnetic) ranges from 300 megahertz to 300 gigahertz. When the source of microwave is switched off, the reactions are stopped immediately. In the presence of argon atmosphere, iridium nanoparticles have been prepared through microwave assisted method. $\quad \operatorname{Ir}_{6}(\mathrm{CO})_{16}$ and 1-butyl-3methylimidazoliumtetraflouroborate have been used as the precursor and reaction medium respectively. Uniformly distributed nanoparticles can be produced through this method.

\subsection{Preparation through Chemical Reduction}

It is one of the common technique to prepare the noble metal based nanoparticles. It is basically referred as a one-step method. Because, the reactants are chemically reduced in a single reactor, and still the desired compound can be achieved. No advance steps/procedure are/is required to separate and purify the intermediate substances. This method helps to produce the desired product in short time. Also, this method involves faster reaction rate.

In one of the work [12], iridium nanoparticles of size between 2.25 and 4.25 nanometers have been prepared by reducing chemically the lithium triethylborohydride. The following chemical substances have been utilized:

- Precursor: $\mathrm{H}_{2} \mathrm{IrCl}_{6}$

- Media: tetrahydrofuran

- Stabilizer: octadecanethiol

The as prepared nanoparticles were found to be crystalline in nature, and exhibit face centered cubic packing. The preparation has been done under ambient atmosphere. In another work [13], iridium nanoparticles of size nearly 3 nanometers have been produced. The method of preparation is chemical reduction. Precursor used is $\mathrm{IrCl}_{3^{-}}$ ethylene glycol. The reduction was very mild, for obtaining the Iridium nanoparticles.

\section{Literature on Nanomaterials/Nanoparticles}

Recently [14], oxide of composition $\mathrm{Li}_{1.2} \mathrm{Mn}_{0.56} \mathrm{Co}_{0.12} \mathrm{Ni}_{0.12} \mathrm{O}_{2}$, has been prepared with distinct morphology. It means that the material synthesized is in the form of micro-sphere/rod, nanoplate and nanoparticle. It was found that the electrochemical properties were completely dependent on the morphology. Also, it was identified that microrods and nanoparticles exhibit high and low electrochemical properties respectively. The high electrochemical performance of microrods was may be due to the Lithium active ion sites originated from porosity of the structures. Also, the contact area between the electrolyte and electrode is very large.

Cobalt quantum dots exhibit good electrocatalyticfeatures. In one of the work [15], cobalt nanocomposite has been prepared. The dimensional size is around 3.2 nanometers. It exhibit the properties that are shown in table 1.

Table.1. Properties of Cobalt Nanocomposite

\begin{tabular}{|c|c|c|c|}
\hline S. No. & Material & Property & Value \\
\hline 1. & & Mass activity & $\begin{array}{c}1250 \\
\mathrm{~A} / \mathrm{g}\end{array}$ \\
\cline { 1 - 1 } 2. & \multirow{2}{*}{$\begin{array}{c}\text { Cobalt } \\
\text { Nanocompos } \\
\text { ite }\end{array}$} & Tafel slope & $\begin{array}{c}37 \\
\mathrm{mV} / \mathrm{dec}\end{array}$ \\
\cline { 4 - 4 } & & Overpotential & $0.37 \mathrm{~V}$ \\
\hline 3. & &
\end{tabular}

Further, the nanocomposite also exhibit large dispersion, enhanced electron transfer mechanism and low dimension. Therefore, cobalt quantum dots distributed on reduced graphene oxide possess exceptional electrocatalytic properties.

Noble and stable metals like gold, silver, iridium, platinum, etc. possess tremendous properties like 


\section{www.rspsciencehub.com}

electrical/optical/mechanical/magnetic properties. Especially at the nanoscale, they are said to be stable, active catalysts, and exhibit corrosion resistance [16-22]. As per the existing literature, the metal based nanoparticles can be used as the catalysts. The chemical characteristics of Iridium nanoparticles is mainly based on composition, and surface morphology. It was identified that, the surface enhanced Raman scattering was studied well for Iridium nanochains than Iridium nanoparticles [23-24].

Nanomaterials based on transition metals and noble metals show versatile chemical properties. Mudusu and others [25], have developed the Tinsulphide (SnS) nanomaterials with 1dimension. The developed $\mathrm{SnS}$ nanostructures high single crystalline nature, and exhibit growth ( $\left.\begin{array}{lll}1 & 0 & 0\end{array}\right)$ plane direction. These nanostructures may be useful for making $\mathrm{SnS}$ based photovoltaics, batteries, etc. The $\mathrm{SnS}$ nanowires preparation is briefly shown, as follows:

- Catalyst: Sapphire, modified by gold

- Substrate: Silicon

- Technique: chemical vapour deposition

- Temperature range: $500-950{ }^{\circ} \mathrm{C}$

- Growth Mechanism: Vapor $\rightarrow$ Liquid $\rightarrow$ Solid

Metal oxide nanoparticles of hollow spheres, have been prepared for the devices of electrochemical energy storage [26-29]. The nanoparticles include nickel oxide $(\mathrm{NiO})$, tungsten oxide $\left(\mathrm{WO}_{3}\right)$, titanium oxide $\left(\mathrm{TiO}_{2}\right)$, etc. The size of $\mathrm{NiO}$ nanomaterials is nearly 28 nanometers. Through hydrothermal method, cobalt oxide $\left(\mathrm{Co}_{3} \mathrm{O}_{4}\right)$ quantum dots have been prepared [30]. The crystallite size of these quantum dots is approximately 2 nanometers. Transition metal based nanostructures are found to be alternate candidates for platinum and other noble metal catalysts. On the titanium foil, $\mathrm{Co}_{3} \mathrm{O}_{4}$ nanomaterial is fully grown. It exhibit rod structure with a sharp tip. On average, the diameter of the rods is 400 nanometers and length is around 10 micrometers. These properties were altered when $\mathrm{Co}_{3} \mathrm{O}_{4}$ was doped with zinc $\left(\mathrm{Zn}^{+2}\right)$.

From the existing literature, we found that, nanoparticles of size $<10$ nanometers can be produced through hydrothermal method. But still, chemical reduction is an effective method to synthesize nanoparticles. Because, the
Volume 02 Issue 12 December 2020

electrochemical properties are high for the nanomaterials prepared through chemical reduction. Cobalt quantum dots have been prepared by dispersing them in graphene nano sheets. The size of the prepared cobalt quantum dots is 3.2 nanometers, on average [15].

In one of the recent work [6], $\mathrm{Cr}$ doped $\mathrm{ZnO}$ nanomaterials have been prepared through hydrothermal route. The XRD analysis indicated that materials exhibit Wurtzite hexagonal structure. The space group of the structure is identified as $\mathrm{P} 36 \mathrm{mc}$. The magnetization values (x) at $27{ }^{\circ} \mathrm{C}$ and $-263{ }^{\circ} \mathrm{C}$ are given in table 2 .

Table.2. Magnetization Values of $\mathrm{Cr}$ doped ZnO, at Different Temperatures

\begin{tabular}{|c|c|c|c|}
\hline S.No. & Material & $\begin{array}{c}\mathbf{X} \text { at 27 } \\
(\mathbf{e m u} / \mathbf{g})\end{array}$ & $\begin{array}{c}\mathbf{X} \text { at } \mathbf{- 2 6 3} \\
(\mathbf{e m u} / \mathbf{g})\end{array}$ \\
\hline 1. & $\mathrm{Zn}_{0.97} \mathrm{Cr}_{0.03} \mathrm{O}$ & 0.038 & 0.40 \\
\hline 2. & $\mathrm{Zn}_{0.95} \mathrm{Cr}_{0.05} \mathrm{O}$ & 0.055 & 0.90 \\
\hline 3. & $\mathrm{Zn}_{0.93} \mathrm{Cr}_{0.07} \mathrm{O}$ & 0.148 & 1.26 \\
\hline
\end{tabular}

The magnitude of field (Coercive field (C)) required to make magnetization zero, and the values of remanant magnetization $\left(\mathrm{M}_{\mathrm{r}}\right)$ is shown table 3 and table 4 respectively.

\section{Table. 3. Magnetization Properties of $\mathrm{Cr}$ doped $\mathrm{ZnO}$, at $100 \mathrm{~K}$}

\begin{tabular}{|c|c|c|c|}
\hline S.No. & Material & $\mathbf{C}(\mathbf{O e})$ & $\mathbf{M}_{\mathbf{r}}(\mathbf{e m u} / \mathbf{g})$ \\
\hline 1. & $\mathrm{Zn}_{0.97} \mathrm{Cr}_{0.03} \mathrm{O}$ & 45 & 0.00030 \\
\hline 2. & $\mathrm{Zn}_{0.95} \mathrm{Cr}_{0.05} \mathrm{O}$ & 126 & 0.0029 \\
\hline 3. & $\mathrm{Zn}_{0.93} \mathrm{Cr}_{0.07} \mathrm{O}$ & 137 & 0.0041 \\
\hline
\end{tabular}

Table. 4. Magnetization Properties of $\mathrm{Cr}$ doped $\mathrm{ZnO}$, at $10 \mathrm{~K}$

\begin{tabular}{|c|c|c|c|}
\hline S.No. & Material & $\mathbf{C}(\mathbf{O e})$ & $\mathbf{M}_{\mathbf{r}}(\mathbf{e m u} / \mathbf{g})$ \\
\hline 1. & $\mathrm{Zn}_{0.97} \mathrm{Cr}_{0.03} \mathrm{O}$ & 49 & 0.00088 \\
\hline 2. & $\mathrm{Zn}_{0.95} \mathrm{Cr}_{0.05} \mathrm{O}$ & 230 & 0.0093 \\
\hline 3. & $\mathrm{Zn}_{0.93} \mathrm{Cr}_{0.07} \mathrm{O}$ & 261 & 0.0121 \\
\hline
\end{tabular}

\section{Conclusions}

Based on the review that has been done in this work, the following conclusions and recommendations have been made: 
- For electrochemical applications, nanomaterials based on transition metals are quite useful.

- Very low size $(<10 \mathrm{~nm})$ nanomaterials can be prepared through the process of sputtering.

- Chemical reduction is mostly used to prepare noble metal based nanomaterials.

\section{Acknowledgement}

We thank the partial financial support of DSTSERB.

\section{References}

[1] Lalitha A. Kolahalam, I.V. Kasi Viswanath, Bhagavathula S. Diwakar, Govindh B, Venu Reddy and Murthy YLN (2019). Review on nanomaterials: Synthesis and applications, Materials Today: Proceedings, 18, 2182-2190.

[2] Risal Singh and ShipraMital Gupta (2016). Introduction to Nanotechnology, Oxford University Press.

[3] Vollath D (2019). Nanomaterials: An Introduction to Synthesis, Properties and Applications, $2^{\text {nd }}$ Edition.

[4] GovindhanMaduraiveeran,

ManickamSasidharan and Wei Jin (2019). Earth-abundant transition metal and metal oxide nanomaterials: Synthesis and electrochemical applications, Progress in Materials Science, 106, 100574.

[5] Bryan Ronain Smith and Sanjiv Sam Gambhir (2017). Nanomaterials for In Vivo Imaging, Chem. Rev., 117, 901-986.

[6] TanumoyDebnath, AtulBandyopadhyay, TanmoyChakraborthy, Sukhen Das and SoumyadityaSutradhar (2019). Influence of different $\mathrm{Cr}$ concentrations on the structural and ferromagnetic properties of $\mathrm{ZnO}$ nanomaterials prepared by the hydrothermal synthesis, Materials Research Bulletin, 118, 110480.

[7] Sato K and Yoshida HK (2000). Material Design for Transparent Ferromagnets with ZnO-Based Magnetic Semiconductors, Japanese Journal of Applied Physics, 39, L555.

[8] Wang B, Iqbal J, Shan X, Huang G, Fu H, Yu $\mathrm{R}$ and $\mathrm{Yu} \mathrm{D}$ (2009). Effects of Cr-doping on the photoluminescence and ferromagnetism at room temperature in $\mathrm{ZnO}$ nanomaterials prepared by soft chemistry route, Materials Chemistry and Physics, 113, 103-106.

[9] You T, Niwa O, Kurita R, Iwasaki Y, Hayashi K, Suzuki K and Hirono S (2004). Reductive $\mathrm{H} 2 \mathrm{O} 2$ Detection at Nanoparticle Iridium/Carbon Film Electrode and Its Application as L-Glutamate Enzyme Sensor, Electroanalysis, 16, 54-59.

[10]Irfan M, Pham X, Nam Han K, Ai Li C, Hyo Hang M and Hun Seong G (2014). Decoration of carbon nanotube films with iridium nanoparticles and their electrochemical characterization, BioChip Journal, 8, 129-136.

[11]So MH, Ho CM, Chen R and Che CM (2010). Hydrothermal Synthesis of Platinum-GroupMetal Nanoparticles by Using HEPES as a Reductant and Stabilizer, Chemistry An Asian Journal, 5, 1322-1331.

[12]Yee CK, Jordan R, Ulman A, White H, King A, Rafailovich M and Sokolov J (1999). Novel One-Phase Synthesis of Thiol-Functionalized Gold, Palladium, and Iridium Nanoparticles using Superhydride, Langmuir, 15, 3486-3491.

[13]Bonet F, Delmas V, Grugeon S, Herrera Urbina R, Silvert P-Y and Tekaia-Elhsissen K (1999). Synthesis of monodisperse Au, Pt, Pd, $\mathrm{Ru}$ and Irnanoparticles in ethylene glycol, Nanostructured Materials, 11, 1277-1284.

[14]Fang Fu, Yuze Yao, Haiyan Wang, Xu GL, Amine K, Sun SG and Minhua Shao (2017). Structure dependent electrochemical performance of $\mathrm{Li}$-rich layered oxides in lithium-ion batteries, Nano Energy, 35, 370378.

[15]Govindhan M, Brennan Mao and Aicheng Chen (2016). Novel cobalt quantum dot/graphene nanocomposites as highly efficient electrocatalystsfor water splitting, Nanoscale, 8, 1485-1492.

[16]Tobias Reier, MehtapOezaslan and Peter Strasser (2012). Electrocatalytic Oxygen Evolution Reaction (OER) on $\mathrm{Ru}$, Ir, and $\mathrm{Pt}$ Catalysts: A Comparative Study of Nanoparticles and Bulk Materials, ACS Catal., 2, 1765-1772.

[17]Karan HI, Sasaki K, Kuttiyiel K, Farberow CA, Mavrikakis M and Adzic RR (2012). Catalytic Activity of Platinum Monolayer on Iridium and Rhenium Alloy Nanoparticles for 
the Oxygen Reduction Reaction, ACS Catal., 2, 817-824.

[18]Fan GY, Zhang L, Fu HY, Yuan ML, Li RX, Hua Chen and Li XJ (2010). Hydrous Zirconia supported iridium nanoparticles: An excellent catalyst for the hydrogenation of haloaromatic nitro compounds, Catalysis Communications, 11, 451-455.

[19]In Soo Park, Min Serk Kwon, Kyung Yeon Kang, Jae Sung Lee and Jaiwook Park (2007). Rhodium and Iridium Nanoparticles Entrapped in Aluminum Oxyhydroxide Nanofibers: Catalysts for Hydrogenations of Arenes and Ketones at Room Temperature with Hydrogen Balloon, Advanced Synthesis and Catalysis, 349, 2039-2047.

[20]Redel E, Kramer J, Thomann R and Janiak C (2009). Synthesis of Co, Rh and Ir nanoparticles from metal carbonyls in ionic liquids and their use as biphasic liquid-liquid hydrogenation nanocatalysts for cyclohexene, Journal of Organometallic Chemistry, 694, 1069-1075.

[21]Kiran V, Ravikumar T, Kalyanasundaram NT, Krishnamurthy S, Shukla AK and Sampath S (2010). Electro-Oxidation of Borohydride on Rhodium, Iridium, and Rhodium-Iridium Bimetallic Nanoparticles with Implications to Direct Borohydride Fuel Cells, Journal of the Electrochemical Society, 157, B1201.

[22]Zhu Yinghuai, KohChenyan, AngThiam Peng, Emi A, Monalisa W, Louis LKJ, Hosmane NS and Maguire JA (2008). Inorg. Chem., 47, 5756-5761.

[23]Malin Cui, Yuan Zhao, Chan Wang and Qijun Song (2016). Synthesis of $2.5 \mathrm{~nm}$ colloidal iridium nanoparticles with strong surface enhanced Raman scattering activity, MicrochimicaActa, 183, 2047-2053.

[24]Chakrapani K and Sampath S (2014). Spontaneous assembly of iridium nanochainlike structures: surface enhanced Raman scattering activity using visible light, Chemical Communications, 50, 3061-3063.

[25]Mudusu D, Koteeswara Reddy N, Sreekantha Reddy D, Jang Won Kang, Sung Ha Park and Charles TW (2017). Growth of singlecrystalline cubic structured tin(II) sulfide ( $\mathrm{SnS}$ ) nanowires by chemical vapor deposition, RSC Advances, 7, 41452-41459.
[26]Sasidharan M, Gunawardhana N, Senthil C and Yoshio M (2014). J. Mater. Chem. A, 2, 7337-7344.

[27]Sasidharan M, Gunawardhana N, Yoshio M and Nakashima $\mathrm{K}$ (2012). $\mathrm{WO}_{3}$ hollow nanospheres for high-lithium storage capacity and good cyclability, Nano Energy, 1, 503508.

[28]Sasidharan M, Nakashima K, Gunawardhana N, Toshiyuki Yokoi, Masamichi Inoue, ShinichiYusa, Masaki Yoshio and Takashi Tatsumi (2011). Novel titania hollow nanospheres of size $28 \pm 1 \mathrm{~nm}$ using soft-templates and their application for lithium-ion rechargeable batteries, Chemical Communications, 47, 6921-6923.

[29]ManickamSasidharan and Kenichi Nakashima (2014). Core-Shell-Corona Polymeric Micelles as a Versatile Template for Synthesis of Inorganic Hollow Nanospheres, Acc. Chem. Res., 47, 157-167.

[30]Guangxing Zhang, Jie Yang, Han Wang, Haibiao Chen, Jinlong Yang and Feng Pan (2017). $\mathrm{Co}_{3} \mathrm{O}_{4-\delta}$ Quantum Dots As a Highly Efficient Oxygen Evolution Reaction Catalyst for Water Splitting, ACS Appl. Mater. Interfaces, 9, 16159-16167. 